\title{
POINTWISE CONVERGENCE AND CESÀRO SUMMABILITY OF DOUBLE ORTHOGONAL SERIES
}

\author{
FERENC MóRICZ AND ZoLtÁn NÉMETH
}

Abstract. Let $(X, \mathscr{F}, \mu)$ be a positive measure space and $\left\{\phi_{j, k}(x): j, k=1,2, \ldots\right\}$ be a double orthonormal system of real-valued functions on $X$. We extend four previous results of Borgen [2] and Tandori [4, 5] from single to double orthogonal series.

Mathematics subject classification (2010): 42B08, 42C10.

Keywords and phrases: Double orthogonal series, pointwise convergence, Cesàro summability.

\section{REFERENCES}

[1] G. Alexits, Convergence Problems of Orthogonal Series, Pergamon Press, Oxford and New York, 1961.

[2] S. Borgen, Über (C,1)-Summierbarkeit von Reihen orthogonaler Funktionen, Math. Ann., 98 (1928), 125-150.

[3] F. MóRICZ, On the a.e. convergence of the arithmetic means of double orthogonal series, Trans. Amer. Math. Soc., 297 (1986), 763-776.

[4] K. TANDoRI, Über die orthogonalen Funktionen IV. (Starke Summation), Acta Sci. Math. (Szeged), 19 (1959), 18-25.

[5] K. TANDORI, Über die orthogonalen Funktionen VI. (Eine genaue Bedingung für die starke Summation), Acta Sci. Math. (Szeged), 20 (1959), 14-18. 